\title{
ARTIFICIAL REEFS TO IMPROVE AND PROTECT FISHING GROUNDS
}

Juan J. Muñoz-Pérez

Applied Physics Dept., University of Cadiz, CASEM, Rio San Pedro s/n, 11540, Puerto Real, Spain juanjose.munoz@uca.es

\begin{abstract}
Different artificial reefs have been invented in recent times to create or to improve fishing grounds. Nevertheless, the use of harmful illegal trawl equipment has become so prevalent that the protection of over-exploited coastal areas has lead some authors to develop another type of artificial reef specially designed to avoid it. A review of some of the latest patents of two types of artificial reefs for marine life (creative and protective) is presented. These designs have been chosen because they introduce a new shape, material and/or behaviour and will give the readers a wide view of the artificial reefs on offer. Finally, a special design, with anti-trawling purposes, whose use is compatible with open water anchoring zones, is also introduced.
\end{abstract}

Key words: artificial reef, anchoring, fishing grounds, illegal trawling

Short running title : CREATIVE AND PROTECTIVE ARTIFICIAL REEFS 


\section{INTRODUCTION}

To capitalise on the benefits that natural reefs can provide, it is also possible to create artificial reefs that mimic characteristics of natural reefs in order to deliver desirable functions such as coastal defence (e.g. flood protection or erosion control), amenity (e.g. angling, diving, surfing) and marine habitat. This paper focuses on this last function. For the former activities (leisure and coastal protection), Challinor and Hall [1] can be consulted

Generally, an artificial reef is a structure that is installed near the shoreline to create space for spawning and inhabitation of marine life and provide a hiding place for small fish, for the purpose of ensuring aquatic resources of onshore fisheries. Early artificial reefs were built by sinking unusable ships or dropping a ground structure into the sea, thus creating inhabitation space. In order to make inhabitable space more orderly, the artificial reef has been developed through many patents, having different shapes (like hexahedrons, cylinders, etc.) or by using various materials (e.g. concrete, waste tires, steel, etc.).

One of the primary functions of many artificial reefs is to create or to improve fishing grounds. The reefs can concentrate species to allow a more efficient fishing, protect small animals and increase the natural productivity and these are some of the main objectives of their installation. Hence, a large number of artificial reef programs have been developed world wide in recent decades to enhance biological production, for example in Japan, France, the United States of America, Portugal, Italy and Spain. More details altogether with data related to the situation before and after the installation of an artificial reef in a specific area can be consulted at Muñoz-Perez et al. [2].

Nevertheless, to protect any already existing productive zones is more profitable from an economical point of view than constructing a new one. So, the protection of over-exploited coastal areas from trawl fisheries, has lead some authors to develop a second type of artificial reef specially designed to avoid the use of illegal trawl gears.

In this paper, a review of some of the latest patents of two types of artificial reefs (creative and protective) is presented. Finally, a special design with anti-trawling purposes, useful for open water anchoring zones, is also introduced.

\section{REVIEW OF RELEVANT PATENTS}

There are several patents regarding artificial reefs. The following designs have been identified as relevant because they introduce a new shape, material and/or behaviour and will give readers a general view of the artificial reefs on offer.

\subsection{Creative artificial reefs}

Many species of fish can be found in the proximity of artificial reefs. Some of them live regardless of its presence, other species live along upwelling or vortices which are created above it, some live in the spaces between artificial reefs, and furthermore, some live within them. Among these fish, it is known that some species spawn, live, rest, or sleep within the vicinity of an artificial reef. Thus, in order to allow 
the artificial reef to efficiently perform, it must be designed to provide an ecological environment which allows several species of fish to coexist. So, the shape is going to be one of the most important features because it will supply zones with different current velocities, different grades of light "protection for fish with different sizes, etc. As far as material is concerned, many different types can be used. The most important is the use of inert and non-corroding components that will neither harm the marine environment nor rapidly be destroyed by the corrosive effect of salt water. Another characteristic to consider is the possibility of using recycled material as well as the price and cost of their construction and deployment.

\subsubsection{Use of recycled materials}

Some patents have considered the use of recycled material. For example, Streichenberger [3] presented an artificial reef made of one or more threads of automobile tires, partially buried in a sedimentary bottom and partially protruding above it. The artificial reef can be built on land and floated to the site of instalment. It can also be built on a boat and dropped directly onto the seabed.

Krenzler [4] tried to improve the former patent (see Fig. (1)) by means of an artificial reef which provides a habitat for small aquatic life and promotes phytoplankton growth. It includes at least one tubular vehicle tire with a wall defining an exterior and a hollow interior and oppositely situated sidewalls interconnected by a threadwall to provide a substantially U-shaped radial cross section. Moreover, at least one corrodible iron must be inserted in the form of a nail, staple, or other shape. Each insert has a first end extended into the hollow interior, a centre securely positioned through the wall, and a second end extended externally to the exterior. The inserts may have varying iron contents to control the rate of corrosion. The reef may also include a weight, apertures, or additional tires to submerge the reef.

A method for manufacturing an artificial reef based on industrial waste is disclosed by Kim [5]. The industrial wastes include:

- red mud; blast furnace slag;

- the sludge from the factories of refining raw ore;

- broken pieces of glass;

- the stone powders from the anti-pollution facilities equipped in stone extracting spots;

- the slurries from the terminal treatment plants of water and sewage;

- the ashes from the incinerators for household wastes;

- the pulp sludge and slurries from paper mills; the coal ashes from thermoelectric power plants;

- and the side-products produced upon coal mining.

All of them are utilized, alone or in combination with a plasticizer and a mineraliser to construct artificial fishing reefs through pug-milling, moulding and thermal treatment processes. The artificial fishing reefs, through which the industrial wastes are converted into environment-affinitive resources, have the advantage of being economically viable and produce no pollution.

\subsubsection{Concrete modules}

The advantages of concrete are the resistance and similarity to natural stone as well as the capability to be moulded. On the downside its weight and cost can be significant. Clamagirand [6] has invented a type of 
artificial reef, both simple and aesthetic, together with its method of installation. The reef comprises a juxtaposition of generally hexagonal towers of a few meters in diameter (Fig. (2)). Each tower can be assembled in-situ, based on two types of simple concrete modules:

- hexagonal plate modules designed to be set horizontally and comprising one or more vertical poles through holes. On the periphery of each horizontal surface, there must be recesses and protuberances enabling them to be nested;

- wall modules forming braces, shaped like rectangular parallelepipeds, designed to be placed vertically. They must comprise a recess on one of their faces and a male protuberance on the opposite face. So, these pieces are capable of being nested into other protuberances and/or recesses of other wall and plate modules which they separate.

- The modules are assembled to one another by one or more metal tie-rods, tubes or lock rings.

Another artificial reef has been developed by Chui [7] which comprises a reef body and internal auxiliary parts. The reef body is made of concrete plates fixed by fixing elements. Films that can prevent shellfish adhering are placed on the inner and outer surfaces of the concrete plate. The invention is easy to construct, and convenient to transport by a small boat. Because of the films, the shellfish adhering to the reef can be removed easily, thereby prolonging its service life and reducing production cost. The invention can increase the scope of the farm of the bottom on the sea without destroying its ecological balance and landscape.

\subsubsection{Steel modules}

According to Hong [8] concrete is useful because is structurally stable. However, it is difficult to build a multi-storey reef because of its weight. Moreover, only a simple structure, such as a hemispherical or a box-shaped one, is possible. These shapes are insufficient to form a complicated internal space, and the sea may be undestrably polluted due to whitening event. Hong [8] developed a functional steel reef. Furthermore, a reef formed from used tires is advantageous in terms of the recycling of resources, but it is problematic because it is difficult to change the shape thereof, and so the reef does not efficiently conduct its function. Thus, as Hong [8] states, the use of steel has been increasing because is relatively lighter than the concrete one and it is possible to form a complex internal space, thus providing a structure suitable for fish inhabitation.

However, most steel reefs which have been constructed have only used a lattice structure. Thus, only an ecological space created by a lattice structure has been provided. In order to entice a larger variety of fish species, a functional steel reef, which provides a complex internal space and creates upwelling or vortices, has been developed (see Fig. (3)). It is evident that the frequency of contacting and stimulating the tactile sense of fishes increases by providing a shadow effect.

The advantages of this material can be summarised as: 
- a complex shape which encourages both calm and turbulent water flow while avoiding areas of water stagnation.

- a structure which offers a large amount of surface area suitable for colonization by marine life

- a cost effective artificial reef in terms of maximum exposed surface area per dollar invested

- design flexibility through use of modular design components which may be constructed either on the site or at some distance and then rapidly transported and deployed to the desired location

\subsubsection{Use of flexible materials}

Flexible materials, such as plastic, can also be used as Hall states in [9] and [10].

In the first case, Hall [9] explains a method of forming an artificial reef unit through locating a tube of a flexible material divided by walls into an array of compartments running the length of the tube (see Fig. (4)) and being arranged in rows and columns so that the tube has a honeycomb structure. The tube is secured in position on the base using one or more flexible and/or rigid elements attached to a fixed object, for example the base. Moreover, some or all of the compartments have to be partially filled with a filler material. The artificial reef unit may be designed to promote an increase in marine life, or to protect a shoreline or a fixed object such as a harbour wall from damage.

In the second case, Hall [10] presents an invention related to a method of manufacturing a framework having a honeycomb structure with a plurality of compartments running the length of the structure. The method inwolves joining a first sheet of a flexible material to a second sheet along a plurality of join lines to form a first row of compartments (see Fig. (5)), joining a third sheet to the second to form a second row of compartments, joining a fourth sheet to the third sheet, and so on. At least some of the compartments may be closed at a first end or a second end or at both ends of the framework. A further feature of the invention relates to a framework produced by the method. The invention may be used in the manufacture of various structures including mine supports, dam walls, storage packs, road supports, contamination barriers, linings and the like.

Jones et al. [11] designed an improved artificial reef module for creating a feeding and shelter habitat for fish. The module includes a column of vertically or horizontally arranged sheets of corrugated material. The corrugated channels of each sheet run transverse to the adjacent sheet, thereby providing a system of complex channels and passageways within the module. Additionally, the flutes of one sheet may have a different height from the flutes of an adjacent sheet, thereby adding to the complexity of the passage system.

\subsubsection{Resinous material}

Dore [12] has patented an expandable, adaptable aquatic life enhancement device which is assembled quickly and easily on location from variously shaped modular panels using hand tools. In a preferred setup of the present invention, the modular panels are constructed of a resinous non-toxic, non-corrodible material, and are of a size and weight which allows as few as two men to handle the modular panels. The device can be used as a refuge for fish, wherein it may be constructed as a shell and have a plurality of fish water entry-existing holes to allow fish to take refuge therein. It may also be used as an oyster reef, 
wherein external flanges are used to attach the modular panel together, and also provide ledges on which the oysters can grow and develop. Moreover, the device can double as a breakwater.

\subsubsection{No specific material}

The following patents do not specify anything about the material, just about the shape. Moore [13] presented a module with a very special shape for coral reef remediation (see Fig. (6)). It includes a central body having an upper, middle and lower settling plate. Extending from the central body there is a plurality of primary tines which include secondary tines extending from them. There are supporting, stabilizing and space filling tines. The branching of the tines closely replicates the appearance of natural branching coral. Over time the individual modules slowly degrade and allow the tines to break off. Bartou'sky [14] invented an artificial reef that promotes marine growth, marine life, and prevents erosion by providing a unique design and configuration (see Fig. (7)). It is a vertical structure having an enclosed top wall, an opened bottom, and at least three side walls forming an overall geometrical shape. An opening extends through the enclosed top wall and at least one opening extends through each side wall. This design and its configuration provide an optimum mechanism for water to flow and efficient stability. It ultimately prevents damage, roll overs, or the like, even during turbulent weather conditions.

Marinaro [15] designed an artificial reef device forming a habitat for fish populations. It includes closely horizontal slabs arranged above one another and has a highly roughened surface and perforations through which young fish can pass (Fig (8))

In 2005, Engler [16] presented a modular unit intended for use as an artificial reef, which can be placed in stacked structures along the bed of an ocean, bay, or other body of water. It comprises a top wall, a bottom wall, opposed side walls and opposed end walls which are interconnected to form a hollow interior (Fig (9)). Every wall is formed with one or more openings whose position and size are designed to allow access of marine life into the interior of the units. These also permit the passage of sunlight therein, direct the flow of sea water into and through the units in a way which increases stability when placed on the ocean bed, and permit alignment of the holes of one unit with those of another when stacked together.

\subsection{Protective artificial reefs}

\subsubsection{To protect the seashore or natural reef}

A way of reducing damage to natural reefs has been to erect structures between the reef and the shoreline so as to reduce the wave force effect upon natural reefs. These artificial reefs have generally consisted of large steel, stone or concrete barriers. Generally, such artificial reefs have consisted of substantially solid barriers with openings so that seawater can move through with some freedom and yet be slowed so as to reduce the impact upon the natural reef areas. While presently available artificial reefs do provide a means to redirect wave forces and thus lessen the impact on natural reefs, they generally lack appropriate design features which are attractive to sea life for habitation and reproduction. 
For example, Black et al. [17] invented an artificial reef for placement on a floor of the seabed adjacent to shore. The reef includes a base supporting an opposing upper surface that has a first end that in use is directed away from the shore and a second end that is directed towards the shore (Fig. (10)). The reef has a total length to total height ratio of at least 5:1 and the upper surface has a convex shape relative to the base so as to have an overall progressively decreasing positive gradient relative to the surface of the water from the first and second ends. The artificial reef may be used to control the wave breaking intensity over the reef.

In a similar way, De Andrade [18] proposes an artificial reef formation to shape sea waves, constituted of pre-moulded and modulated concrete blocks and hollows, that are set submerged and fixed by its own weight, with variable formats, so the waves break following a form previously determined for sea sports practice (surf, windsurf, etc.).

Artieres et al. [19] patented an invention related to an elongated device consisting of at least one tube comprising a water-permeable inner tubular casing for retaining a filler material. The production method is also claimed (Fig. (11)). This invention also comprises an outer lattice-forming casing (net, grille, mesh, etc.) which surrounds the aforementioned inner casing. The invention is suitable for a dike, a quay, a jetty, a breakwater, a groin, an artificial reef or bank reinforcement.

\subsubsection{To protect fishing grounds}

Meisingset [20] designed an anti-trawling artificial reef which consists of a cylindrical core having means for receiving radial outward-projecting elements in the form of hollow or solid section rods or tubes (Fig. (12))

Dounas [21] has developed an integrated system for the automatic implantation of metal tubes in sedimentary seabeds. This system, detailed in Fig. (13), consists of a mechanism with grabs that can hold a metal tube tightly from its upper part in a vertical position. The grabs also hold a watertight seal, the lower part of a flexible water tube connected to the metal tube. By activating a water pump, pressurized water comes out from the lower end of the metal tube, washing out the sediment particles, gradually creating a narrow hole that allows the lowering of the tube to the desired depth. This depth is controlled by shoes which prevent further penetration of the metal tube when they touch the seabed. When the metal tube reaches the desired depth a special tube release mechanism is activated and the main body of the system with the holding grabs and the shoes as well as the flexible tube are brought back to the vessel's working deck in order to be reused for a new tube implantation. This methodology, in soft bottom substrates, can offer a high level of protection to marine seagrass beds and nursery grounds, from illegal trawling and also an alternative to conventional mooring systems (e.g. concrete blocks, sandbags, embedding anchors, etc.). An example of these conventional blocks is shown in Fig. (14). The weight of these modules was established at $6000 \mathrm{~kg}$ according to the strength imposed by the greater trawling ships. These units were grouped into barriers which were placed at a distance between them sufficient to make the trawling task difficult. Instructions about the positioning of the barriers and the identification of the trawling paths can be obtained in Muñoz-Perez et al. [2]. 


\subsubsection{Compatibility with open water anchoring zones}

The inlets and bays of commercial ports are anchoring zones for ships waiting to moor to the quay, etc. Up to this moment these areas could not be protected against illegal trawling activities due to the obvious incompatibility between artificial reef barriers and the anchoring of ships.

Muñoz-Perez and Naranjo [22] presented an invention which led to anchoring zones being compatible with artificial reefs and also avoiding the illegal trawling. In order to achieve this aim, the artificial reef has to be able to trap the trawling gears but to release anchors if they were caught. This module consists on a reinforced concrete slab (see Fig (15), part A) where metallic rods (B) are embedded to catch illegal trawling fishnets easier. The second part of Fig.(15) shows how anchors can be released due to the inclination of the metallic beams, oriented to the outside, and the distance between two adjacent rods, greater than the bigger dimension of the anchor used.

\section{CURRENT SITUATION \& FUTURE DEVELOPMENTS}

It is apparent from the first part of this paper, that a great variety of artificial reefs has been developed, which can be used for protection against illegal trawling, to modify the shape of the waves or to create and improve fishing grounds. These modifications, which are starting to be studied, can change the steepness of a wave. It seems logical to think on future research as giving the possibility for the alteration of the concentration or dissipation of wave energy and even their use for industrial aims.

It may be thought that some of these fields are mature and little attention will be paid to these in the coming years. Nevertheless, the author believes that future patents will deal with various aspects of applications, possibly into areas not covered so extensively.

Methodologies for positioning reefs are capable of being improved for example. The same applies to the real use (not just hypothetical) of materials coming from the recycling of industrial processes.

Furthermore, an artificial reef model which reconciles the protection against illegal trawling activities on anchoring zones has been obtained. The next step should be to explore designs that encourage the construction of artificial reefs for creating or improving fishing grounds within those areas, probably taking into account coastal defence aspects as well. According to Challinor and Hall [1], this research will provide design knowledge for target habitats and species in order to contribute to biodiversity and local fishery resources, and to promote recreational sea angling and diving.

These multi-functional artificial reefs should be tracked through future patent reviews to learn about their design, planning and construction systems. While there is significant knowledge about structures for coastal defence and artificial reefs to improve and protect fishing grounds, there is little work that draws both these aspects together 


\section{REFERENCES}

[1] Challinor S. and Hall H. Multi-functional artificial reefs scoping study. CIRIA C673 report, London $2008,69 \mathrm{pp}$

[2] Muñoz-Perez JJ, Gutierrez JM and Naranjo JM. Position and monitoring of anti-trawling reefs in the Cape of Trafalgar (Gulf of Cadiz, SW Spain). Bulletin of Marine Science 2000; 67: 761-772

[3] Streichenberger, R.: WO/1993;025764 (1993)

[4] Krenzler, L.M.: WO/1998/042184 (1998)

[5] Kim, H.S.: WO/2000/006314 (2000)

[6] Clamagirand, E.: WO/2001/077443 (2001)

[7] Chui, H.K.: WO/2006:056124 (2006)

[8] Hong, S. I.: WO/2006/080721 (2006)

[9] Hall, A.R.M.: WO/2000/008260 (2000)

[10] Hall, A.R.M.: WO/2004/014643 (2004)

[11] Jones, Barton, G. Greene, Ray, E. Blancher, Eldon, C. II : WO/1993/003229 (1993)

[12] Doré, W.J.: WO/2000/006314 (2000)

[13] Moore, M.D.: WO/2002/068762 (2002)

[14] Bartowski, M.S.: WO/2002/053310 (2002)

[15] Marinaro, J-Y: WO/1997/003554 (1997)

[16] Engler, E.: WO/2005/068728 (2005)

[17] Black, K.P., Mead, S., Mathew, J., McComb, P., J. Kim: WO/2003/060241 (2003)

[18] De Andrade, M.C.: WO/2002/061209 (2002)

[19] Artieres, O., Derache, F., Dunand, M., : WO/2006/114547 (2006)

[20] Meisingset, S.: WO/2000,049859 (2000)

[21] Dounas, C.: European Patent EP0897034 (1999)

[22] Muñoz-Perez, J.J. and Naranjo, J.M.: ES 2176052 B1 (2002) 


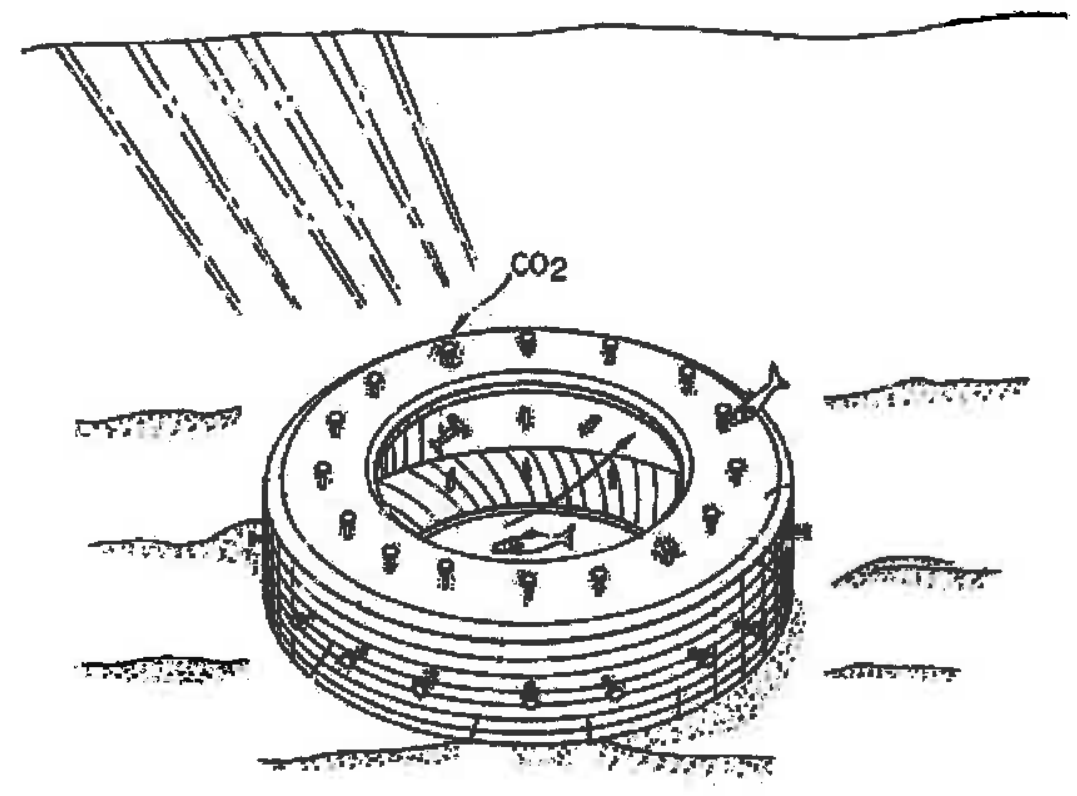

Fig. 1 Krentzler's design [3]

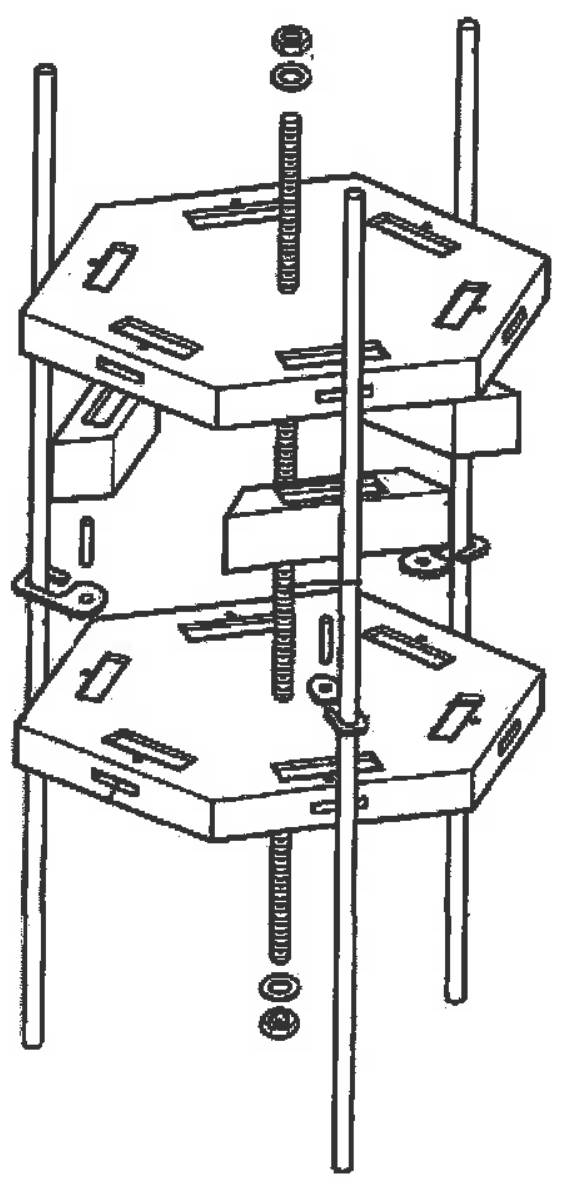

Fig. 2 Clamagirand's design [5]: juxtaposition of hexagonal towers a few meters in diameter 


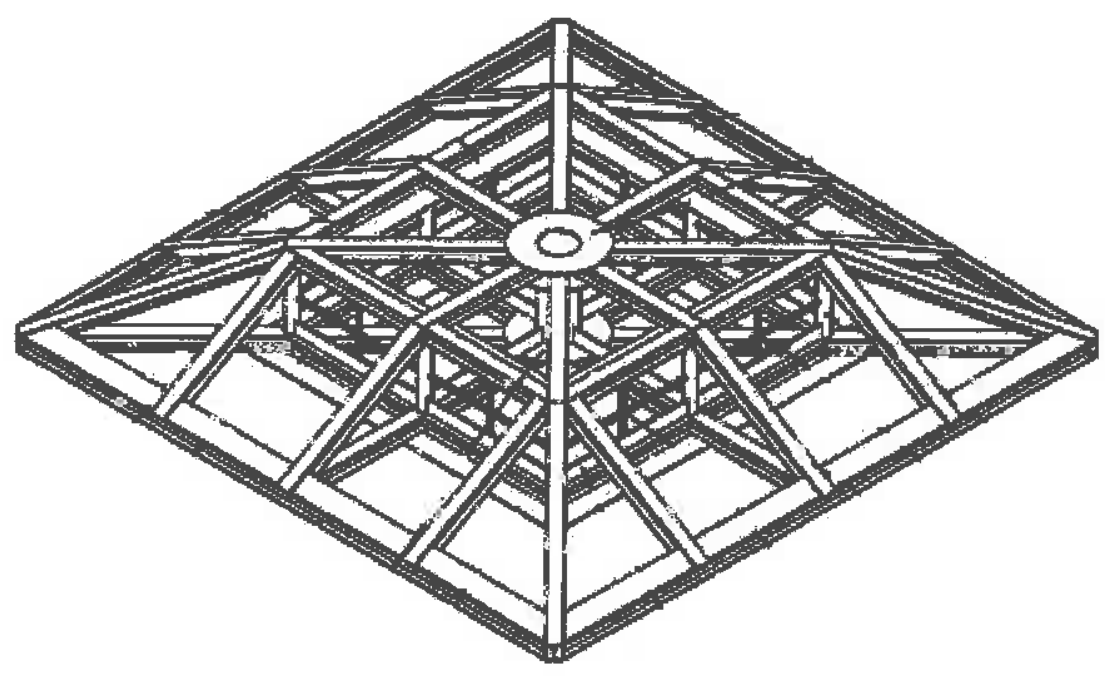

Fig. 3 Hong's design [7]: A steel reef which provides a complex internal space and upwelling or vortices

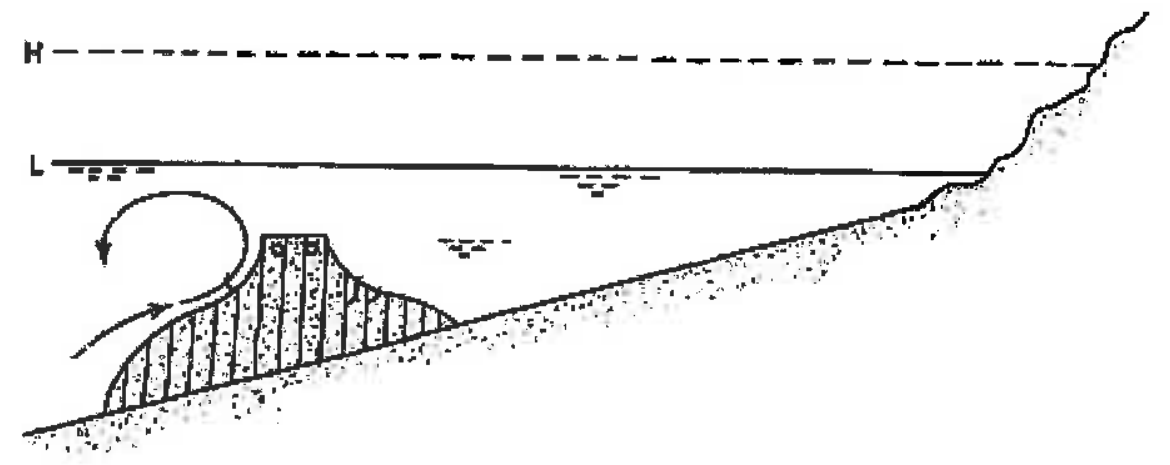

Fig. 4 Hall's design [8]: reef unit through locating a tube of a flexible material divided by walls into an array of compartments 


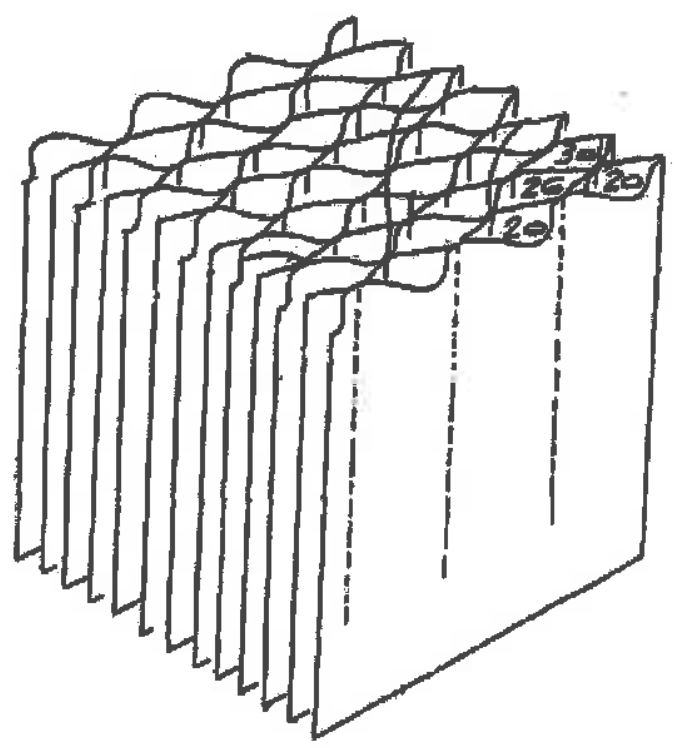

Fig. 5 Another Hall's device [9]: joining sheets of a flexible material along a plurality of join lines to form a succesive rows of compartments

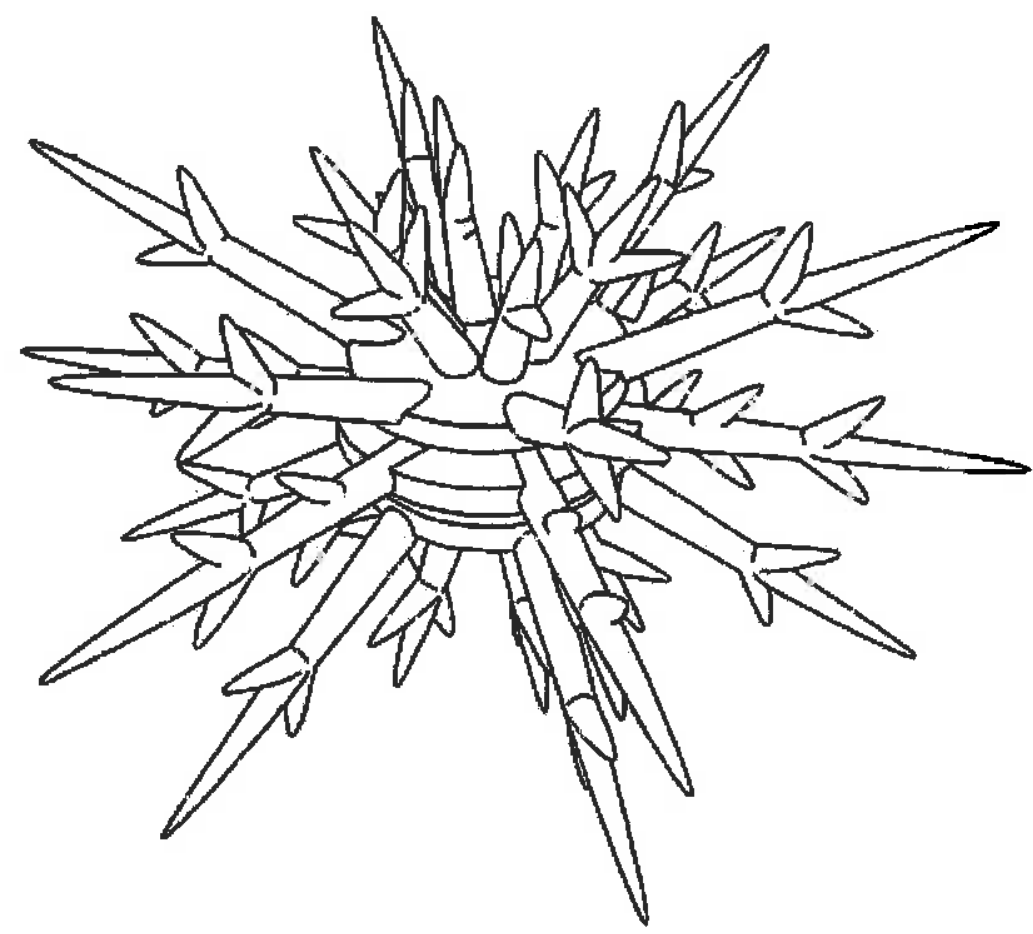

Fig. 6 Moore [12] designed a reef unit with plenty of tines whose branching closely replicates the appearance of natural branching coral. 


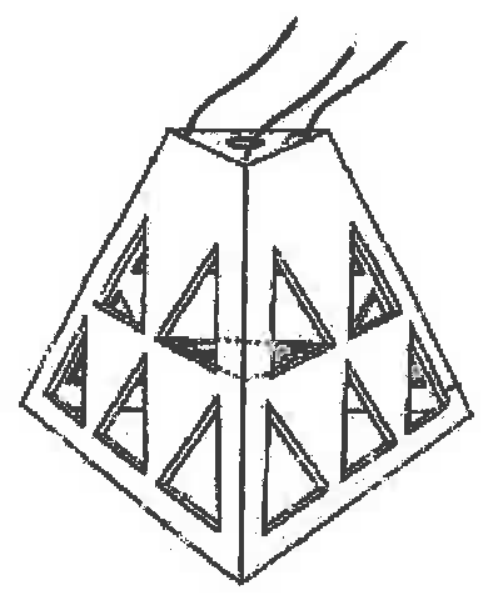

Fig. 7 Bartowsky's structure [13]

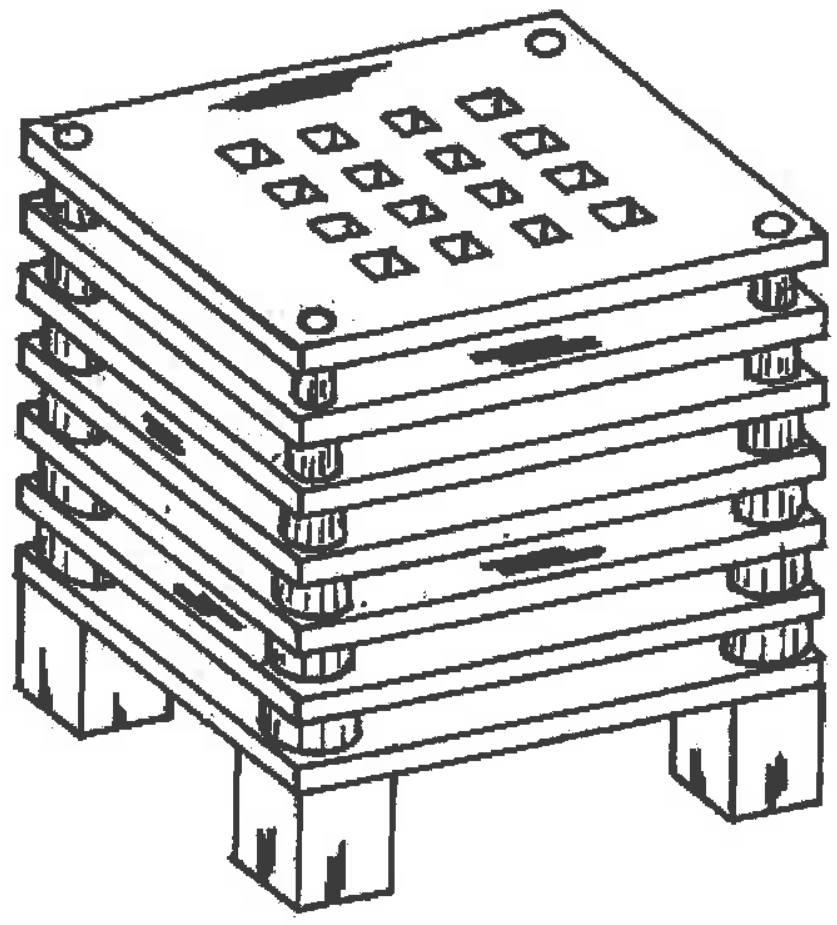

Fig. 8 Marinaro's device including closely spaced horizontal slabs [14] 


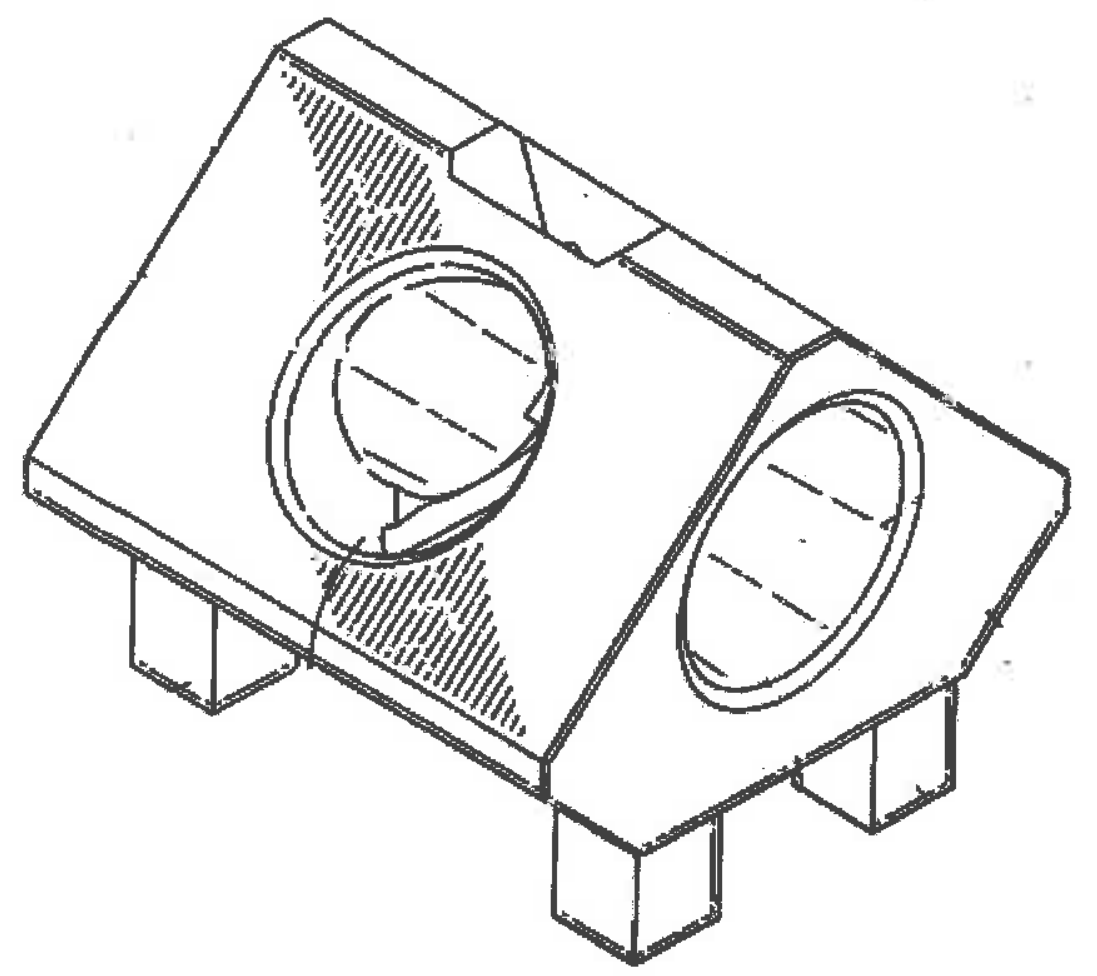

Fig. 9 Engler's reef unit [15]

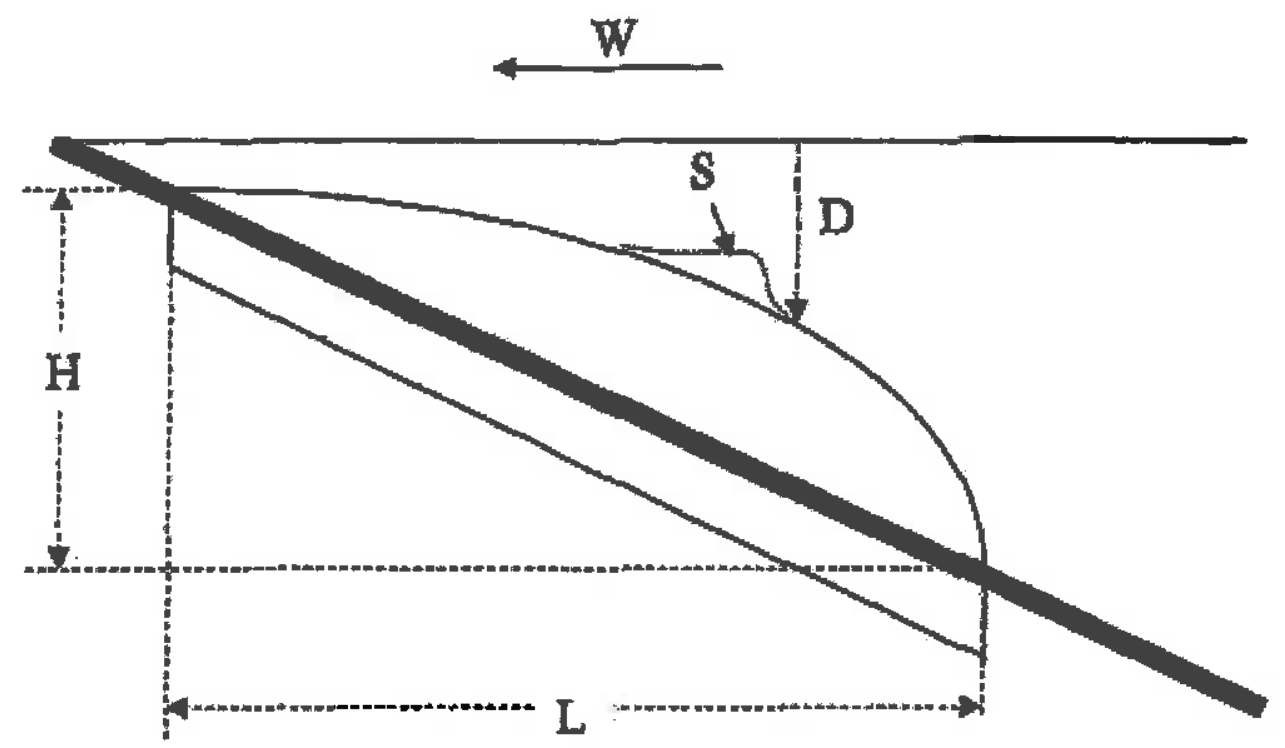

Fig. 10 Black et al. [16] invention: an artificial reef to control the wave breaking intensity 


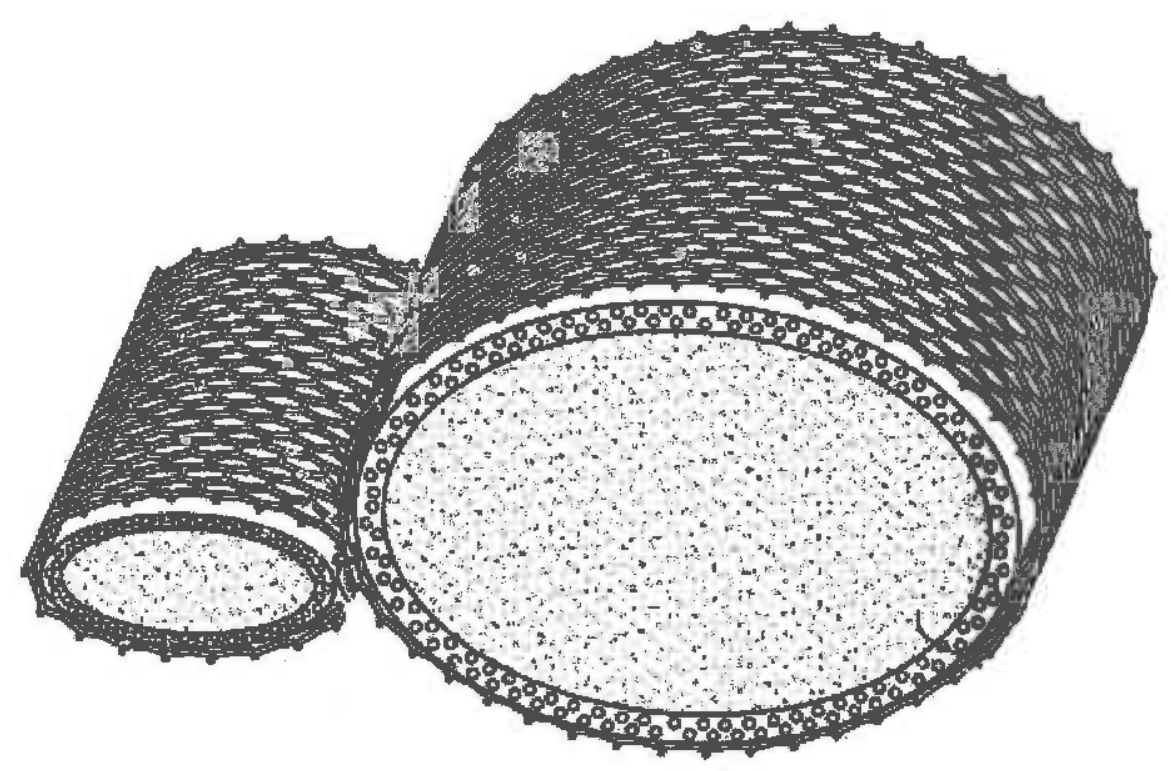

Fig. 11 Artieres et al. [18] invention, consisting of at least one tube comprising a water-permeable inner tubular casing for retaining a filler material

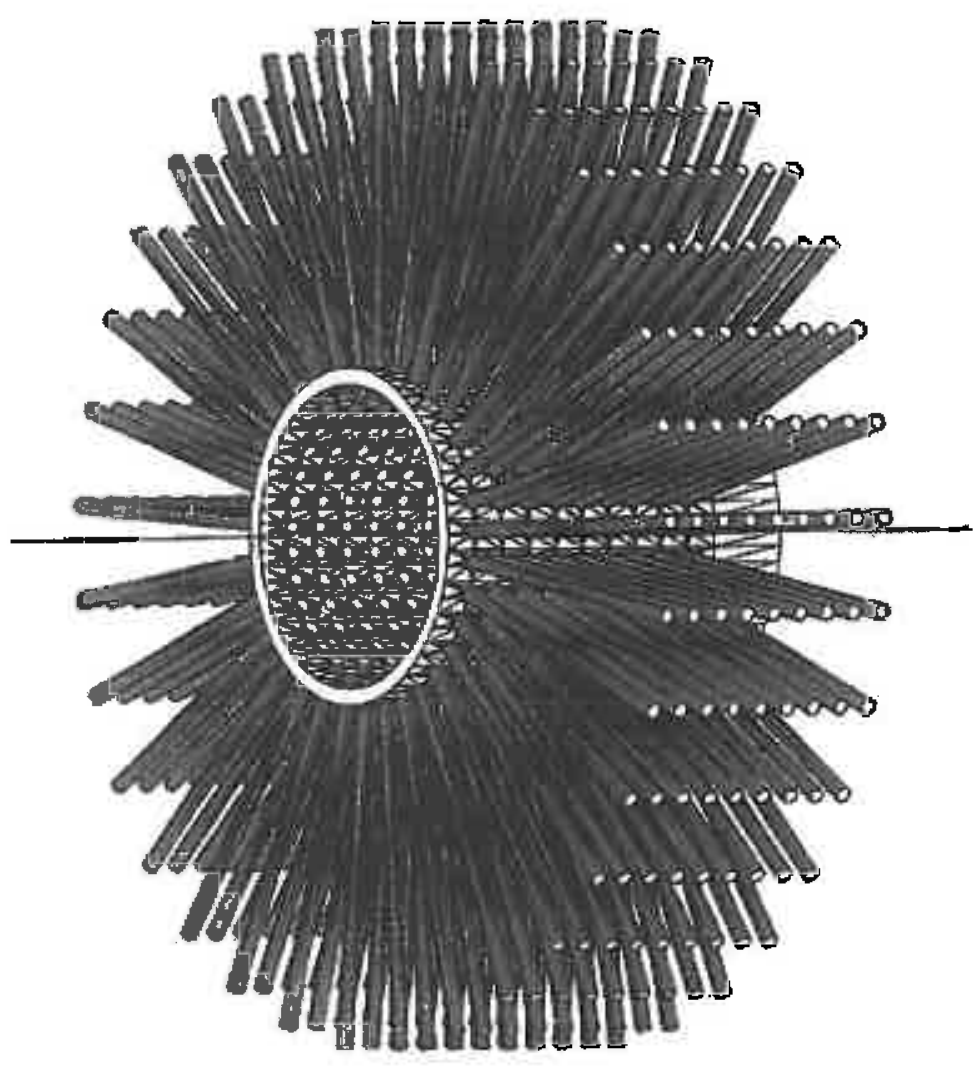

Fig. 12 Meisingset [19]: anti-trawling artificial reef which consists of a cylindrical core with radially outward-projecting elements 


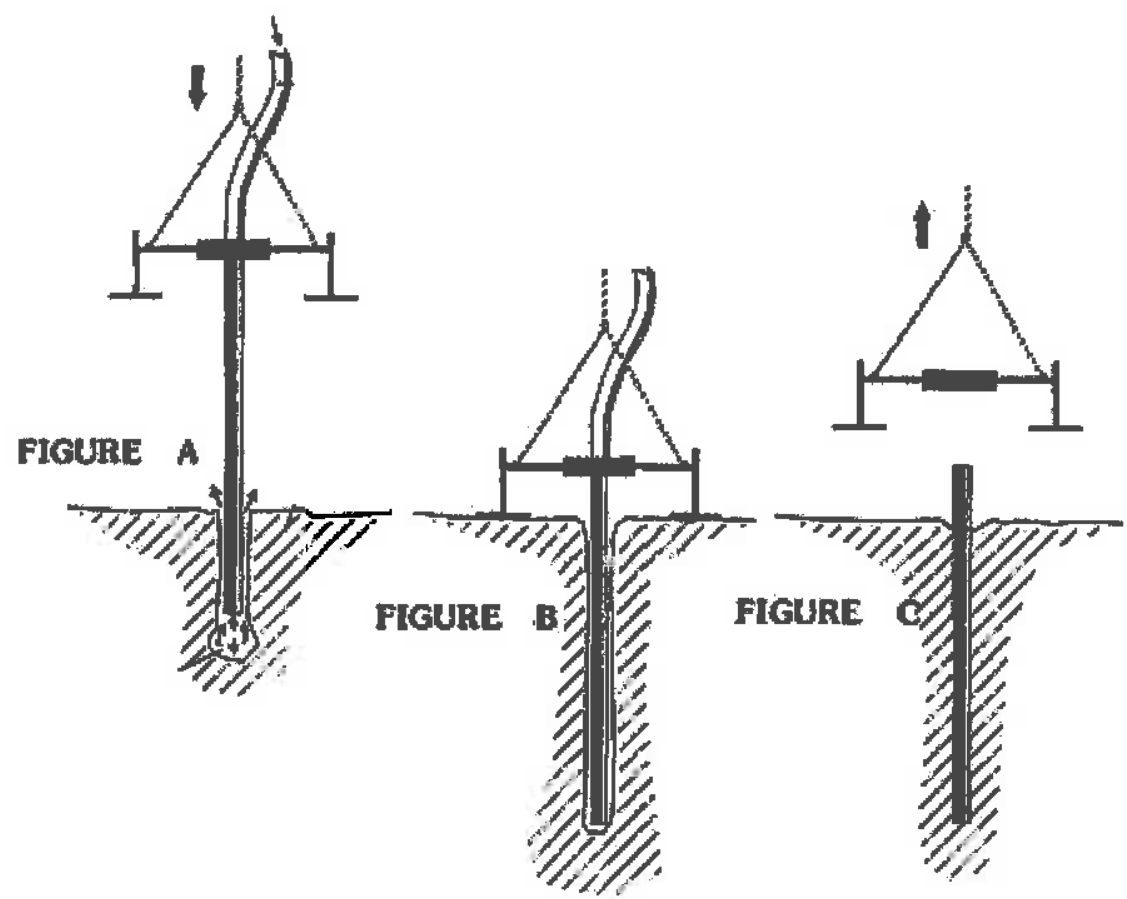

Fig. 13 Dounas [20] device: integrated system for the automatic implantation of metal tubes in sedimentary seabeds

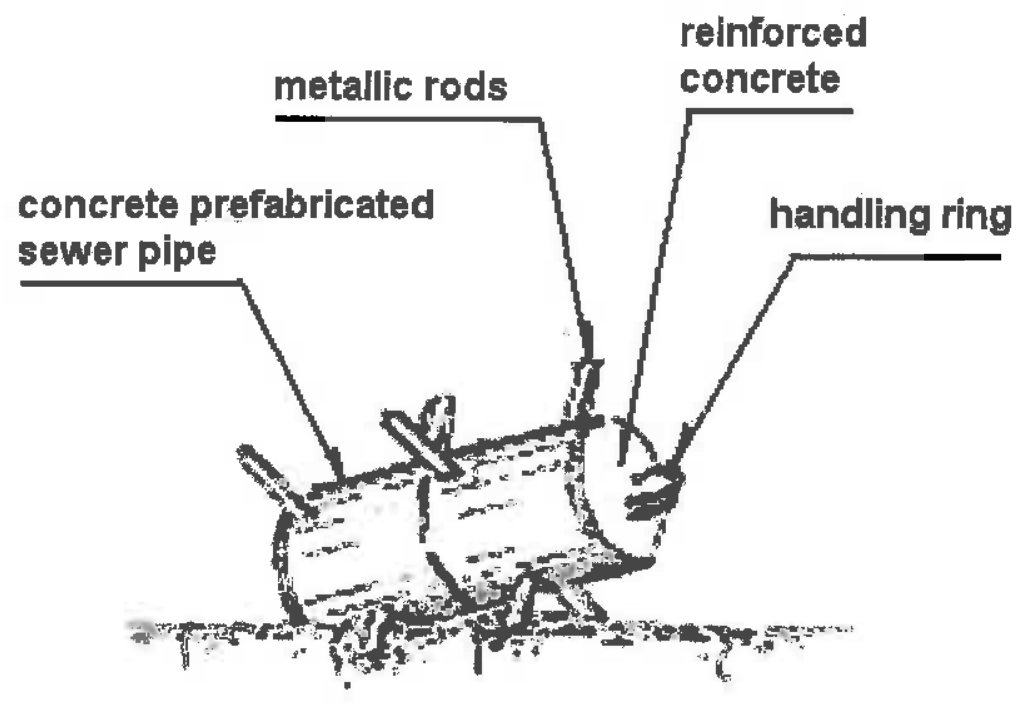

Fig. 14 Anti-trawling conventional block 

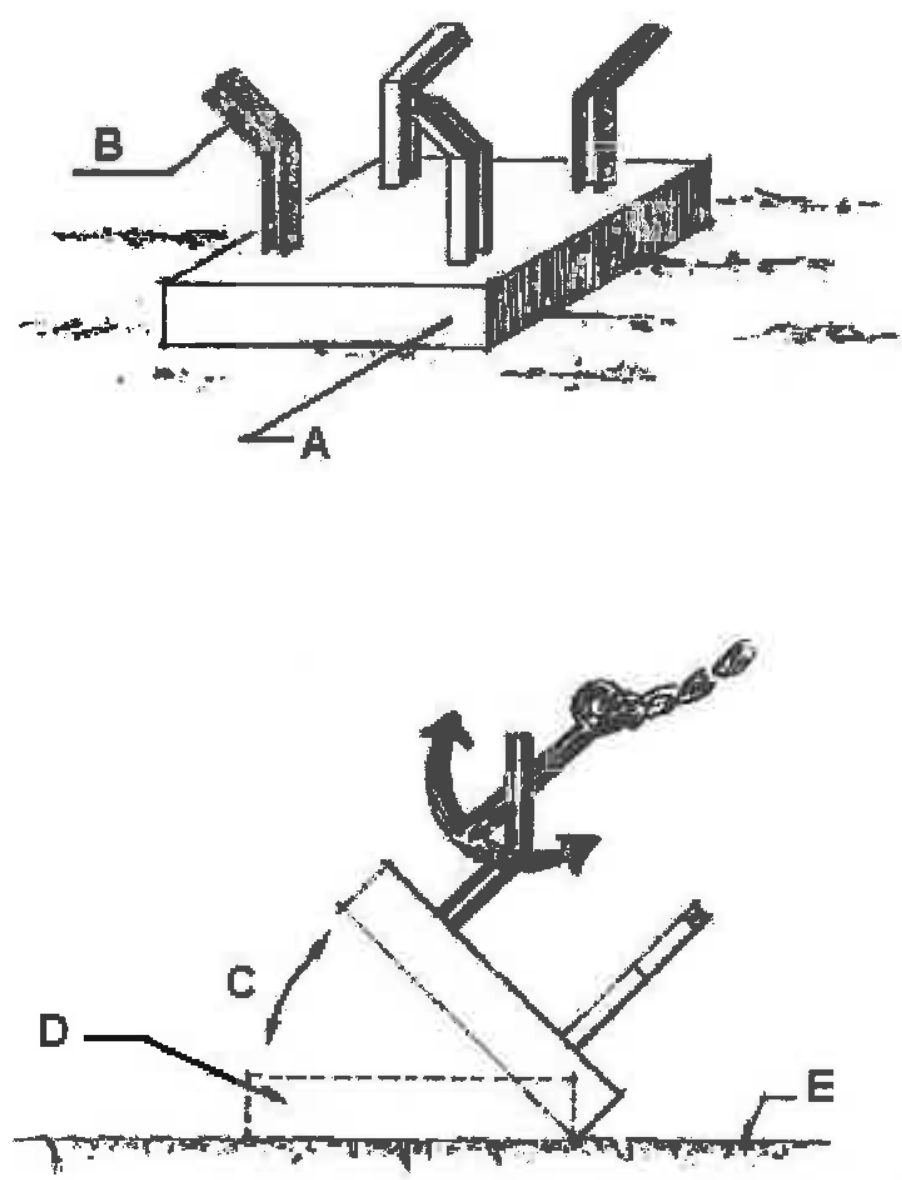

Fig. 15 Muñoz-Perez and Naranjo [21] : artificial unit which let to project anchoring zones compatible with avoiding the illegal trawling. This module consists in a reinforced concrete slab (A) where some metallic rods (B) are embedded. The second sketch shows how the anchor is relieved 